\title{
Medindo o poder refrativo de lentes de contato gelatinosas personalizadas
}

\author{
Measuring the refractive power of customized soft contact lenses
}

\author{
Luis Alberto Vieira de Carvalho ${ }^{1}$ \\ Geunyoung Yoon ${ }^{2}$
}

O presente trabalho foi realizado no Center for Visual Science, University of Rochester - Rochester - USA.

${ }^{1} \mathrm{PhD}$ em óptica e Coordenador de pesquisas do Laboratório de Optica Oftálmica do Instituto Física de São Carlos. Universidade de São Paulo - USP - São Carlos (SP) Brasil. Pos-doc na University of Califórnia at Berkeley e no Center for Visual Science em Rochester, Nova York. ${ }^{2} \mathrm{PhD}$ pela Universidade de Tókio, Japão. Professor Assistente do Center for Visual Science em Rochester, Nova York.

Endereço para correspondência: Grupo de Óptica, Instituto de Física de São Carlos - Universidade de São Paulo. Av. Trabalhador Sãocarlense, 400 - Cx. Postal 369 - São Carlos (SP) CEP 13560-970

E-mails: lavcf@idsc.usp.br

Recebido para publicação em 09.10.2005

Última versão recebida em 05.08 .2006

Aprovação em 07.08.2006

Nota Editorial: Depois de concluída a análise do artigo sob sigilo editorial e com a anuência dos Drs. Paulo Ricardo de Oliveira e Fábio Henrique Cacho Casanova sobre a divulgação de seus nomes como revisores, agradecemos sua participação neste processo.

\begin{tabular}{|c|}
\hline RESUMO \\
\hline 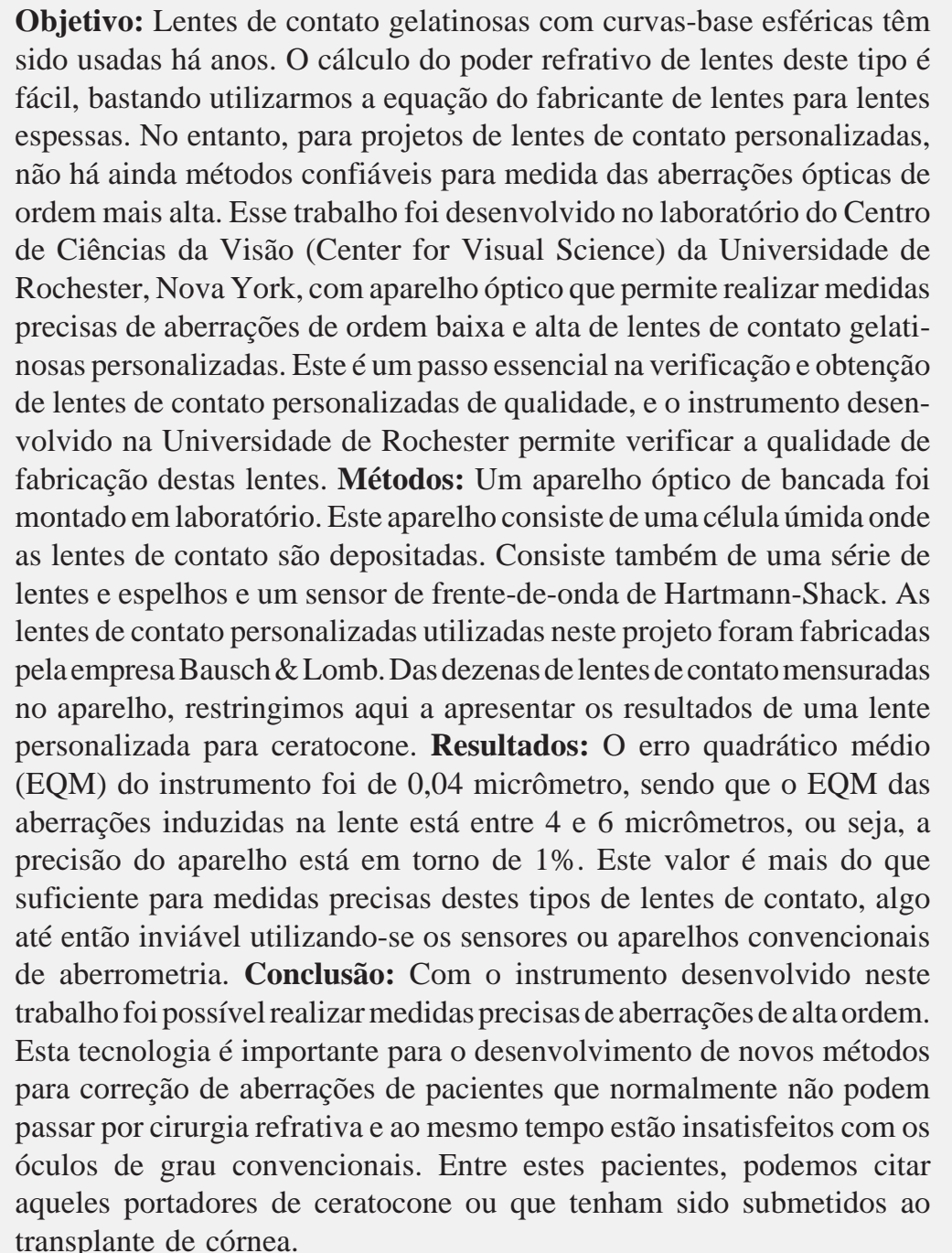 \\
\hline
\end{tabular}

Descritores: Topografia da córnea; Lentes intra-oculares; Refração ocular; Lentes de contato hidrofilicas; Ceratocone

\section{INTRODUÇ̃̃̃O}

Lentes de contato (LC) gelatinosas são utilizadas há décadas ${ }^{(1)}$. Usualmente, estas lentes proporcionam um maior conforto e fluxo lacrimal ${ }^{(2-3)}$ quando comparadas às lentes rígidas. Até recentemente, para se receitar 
uma lente de contato, tinha-se apenas a opção de calcular a curva posterior e anterior de acordo com o índice de refração do material, a correção esférica desejada e a curvatura média do olho do paciente ${ }^{(1-2)}$. Depois disso alguns fabricantes introduziram a possibilidade da correção dos componentes esfero-cilíndricos. Apesar destes dois tipos de lente (para correção apenas do componente esférico e para correção do componente esférico e cilíndrico) solucionarem o principal defeito visual da maioria da população, há certos casos específicos em que o paciente não se adapta. Esta falta de adaptação normalmente ocorre por dois motivos: primeiro, a curvatura da córnea deste paciente é anormal, sendo bastante diferente de uma superfície esférica ou parabólica, como a maioria das córneas normais são; segundo, como a córnea é a principal superfície refrativa do olho, as irregularidades desta induzem aberrações ópticas de alta ordem, como coma e aberração esférica, que não são corrigidas por simples lentes esféricas ou esfero-cilíndricas. Podemos citar, como exemplos destes casos, pacientes com ceratocone ou pacientes que tenham sido submetidos a transplante de córnea. Em ambos casos, o paciente tem uma superfície corneana anterior bastante irregular, gerando problemas ópticos mais complexos do que simples miopia/hipermetropia e/ou astigmatismo. Os problemas ópticos nestes olhos são normalmente uma combinação de diversos outros problemas, que podem ser separados em descritores mais simples, mas que se manifestam de maneira sobreposta, tornando sua correção bem mais complexa do que em casos comuns.

Somente recentemente, com o advento de técnicas de medida usando o sensor de Hartmann-Shack, técnicas de processamento de imagens para detecção dos movimentos de translação e rotação da $\mathrm{LC}^{(3)}$, e técnicas de fabricação com tornos ópticos de alta precisão, é que foi possível vislumbrar a fabricação de lentes de contato personalizadas (LCP) para pacientes que não se adaptam às LC convencionais. Com o advento desta possibilidade, da qual Carvalho, Yoon são pioneiros a necessidade de técnicas mais precisas para medir as lentes de contato, tornouse necessária ${ }^{(4)}$. No entanto, para que estas correções sejam otimizadas, necessitam-se de técnicas suficientemente precisas. Neste trabalho vimos demonstrar um aparato óptico/mecânico que foi desenvolvido com o objetivo de realizar medidas precisas sem a necessidade do paciente estar usando a LCP.

\section{MÉTODOS}

Um sistema óptico de bancada foi construído (Figura 1A). Para realizar as medidas, a LCP é posicionada na célula úmida com a parte côncava voltada para baixo. O diagrama também mostra o trajeto da luz laser $(635 \mathrm{~nm})$ do plano da lente de contato (objeto) até o sensor de Hartmann-Shack (plano imagem) e também, ao passar pelo divisor de feixes, até o foco da câmera da pupila. Esta câmera é essencial para o correto posicionamento da LCP dentro da célula úmida, de tal maneira que esteja corretamente centralizada e corretamente alinhada.

A lente é introduzida pela parte superior da célula úmida, confeccionada em acrílico (Figura 1B), que é aberta e é também por onde se insere o líquido com a solução para LCP. A LCP segue então o caminho abaixo por efeito da gravidade até acomodar-se ao fundo da célula. Para que a medida seja fiel ao formato original da LCP, esta deve estar a mais relaxada possível. Isto é conseguido deixando-se que a LC desça até ao fundo da célula úmida, na posição mais confortável e natural para a LCP, sem influência de ação externa no formato de sua superfície.

Neste processo todo de se posicionar a lente um dos itens mais importantes é evitar a deformação da superfície no momento da medida. Para isso utilizamos uma pinça plástica para

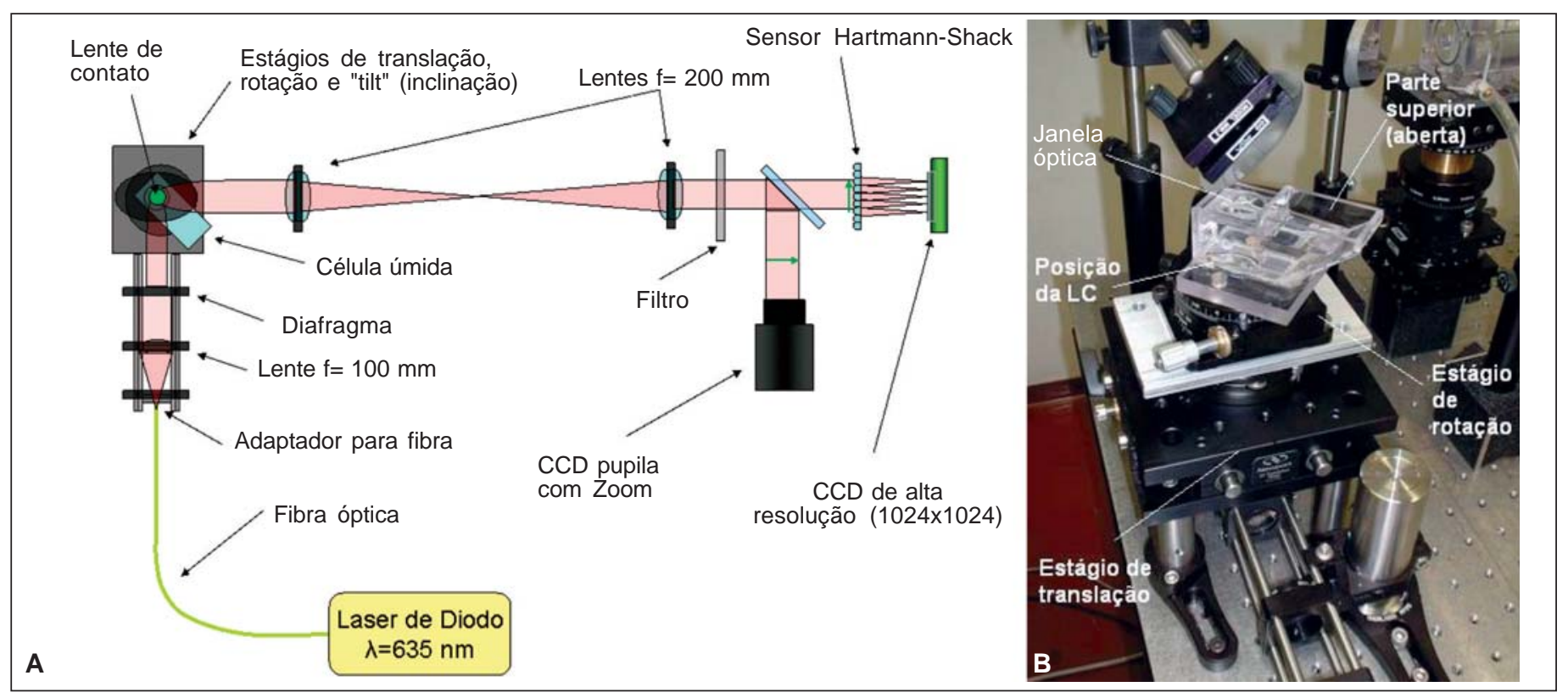

Figura 1 - A: Diagrama óptico do aparelho para medidas de aberrações ópticas em LCP; B: Fotografia do sistema montado na bancada. 
"empurrar" a lente com pequenos toques, de tal maneira que a lente bate em uma das paredes da célula e volta suavemente, flutuando, até a posição natural. Ou seja, a lente fica deitada numa posição próxima àquela que ela ficaria no olho, com poucas deformações, (obviamente, no olho há pressões agindo na lente, que podem causar sua deformação) e assim podemos realizar medidas precisas. Para este processo crítico de alinhamento é fundamental a câmera da pupila mostrada na figura $1 \mathrm{~A}$, onde a imagem da lente pode ser visualizada (Figura 2).

A imagem mostrada na figura 2 é de uma das LCP, já com a zona óptica moldada de maneira personalizada, como pode ser visto na região central da lente (artificialmente circulada em preto). Nesta imagem também podemos ver os pontinhos feitos com marcador cirúrgico para que a posição (translação vertical, horizontal e rotação) da LCP sobre olho possa ser detectada.

Depois de corretamente alinhada e centralizada, são coletadas imagens do sensor de Hartmann-Shack. Estas imagens são então analisadas e processadas pelo programa de computador (Figura 3). Este programa permite o cálculo em tempo real

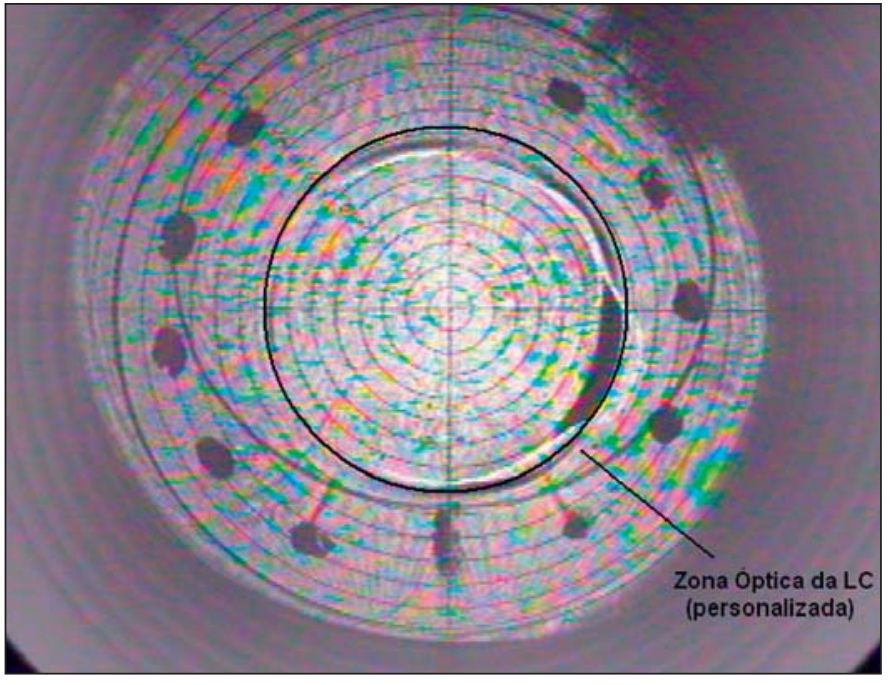

Figura 2 - Imagem da lente de contato feita pela câmera da pupila (mostrada no diagrama óptico da Figura 1A). Esta é uma imagem superior da célula, de tal maneira que se pode fazer um alinhamento e focalização da lente durante o processo de medida.

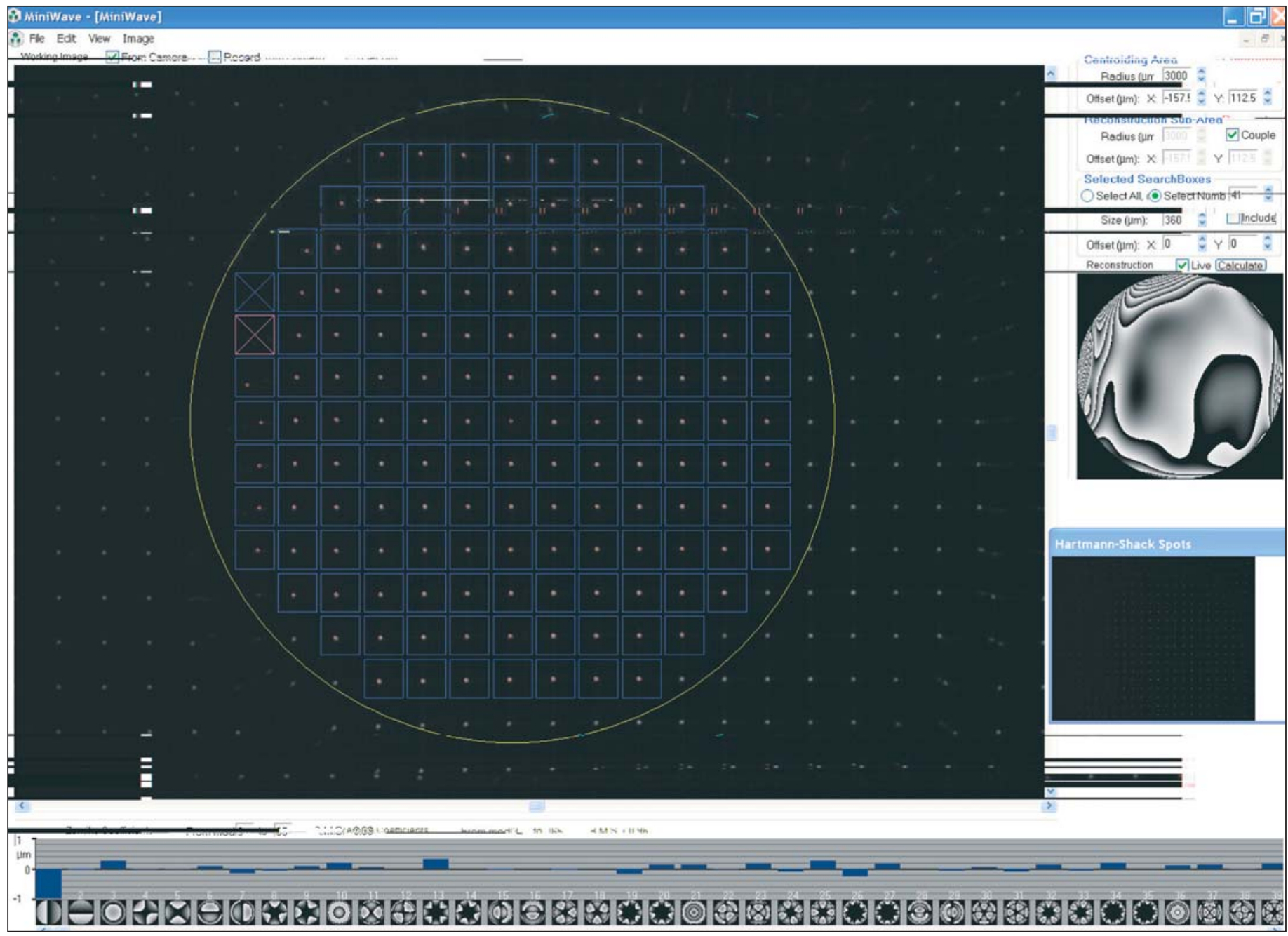

Figura 3 - Tela do software utilizado para processamento das imagens de Hartmann-Shack ${ }^{5}$. Como podemos ver, há vários recursos no programa, dentre eles: possibilidade de escolha do diâmetro da pupila, visualização em tempo real de cada coeficiente de Zernike e da frente de onda, entre outros. 
da frente de onda e também dos primeiros 66 termos de Zernike. Quando a medida é satisfatória, captura-se a imagem e pode-se então exportar os dados dos coeficientes para um outro programa, como uma planilha de cálculo, por exemplo.

A LCP utilizada neste trabalho foi confeccionada para um paciente com ceratocone, pela empresa Bausch \& Lomb em um torno de alta precisão, com algoritmos e técnicas que são de propriedade desta empresa e que não foram divulgados. $\mathrm{O}$ processo de fabricação é baseado em uma série de coeficientes de Zernike que foram enviados para a Bausch \& Lomb (veja Figura 5).

A escolha deste paciente para demonstração do aparelho é por motivos óbvios, ou seja, para este paciente o erro quadrático médio (EQM) da LCP é maior do que para pacientes normais, e isso nos indica claramente que, se a precisão for aceitável para a LCP deste paciente, para pacientes normais as medidas serão ainda mais precisas.

\section{RESULTADOS}

Para demonstrar aqui a qualidade das medidas serão mostrados os resultados para um paciente com ceratocone, o qual obteve excelente benefício visual utilizando-se esta LCP (aberrações de ordem alta para este paciente caíram de 4 micrômetros para 0,5 micrômetro com relação ao erro quadrático médio (EQM)) do que suficiente para o projeto, fabricação e averiguação da qualidade de LCP. Na tabela 1 podemos ver os coeficientes de Zernike relativos a três medidas consecutivas de uma das LCP fabricadas para um de nossos pacientes com ceratocone, e na figura 4 podemos ver um gráfico de barras destes valores. É importante observar que, por motivos didáticos, não foram mostradas as aberrações de primeira e segunda ordem pois estas não são o objetivo das correções deste projeto. Como foi citado anteriormente, para correção de miopia/hipermetropia e astigmatismo já existem lentes esfero-ci-

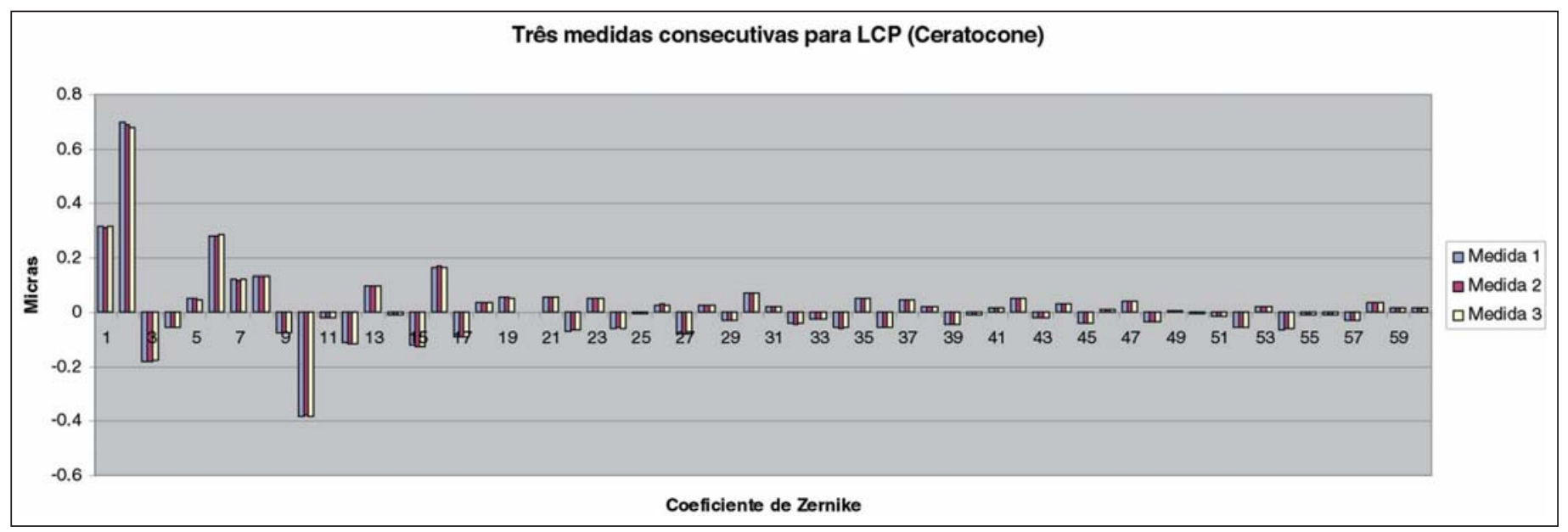

Figura 4 - Resultados dos coeficientes de Zernike para três medidas independentes da LCP do paciente com ceratocone, mostrando os valores individuais para todos os coeficientes

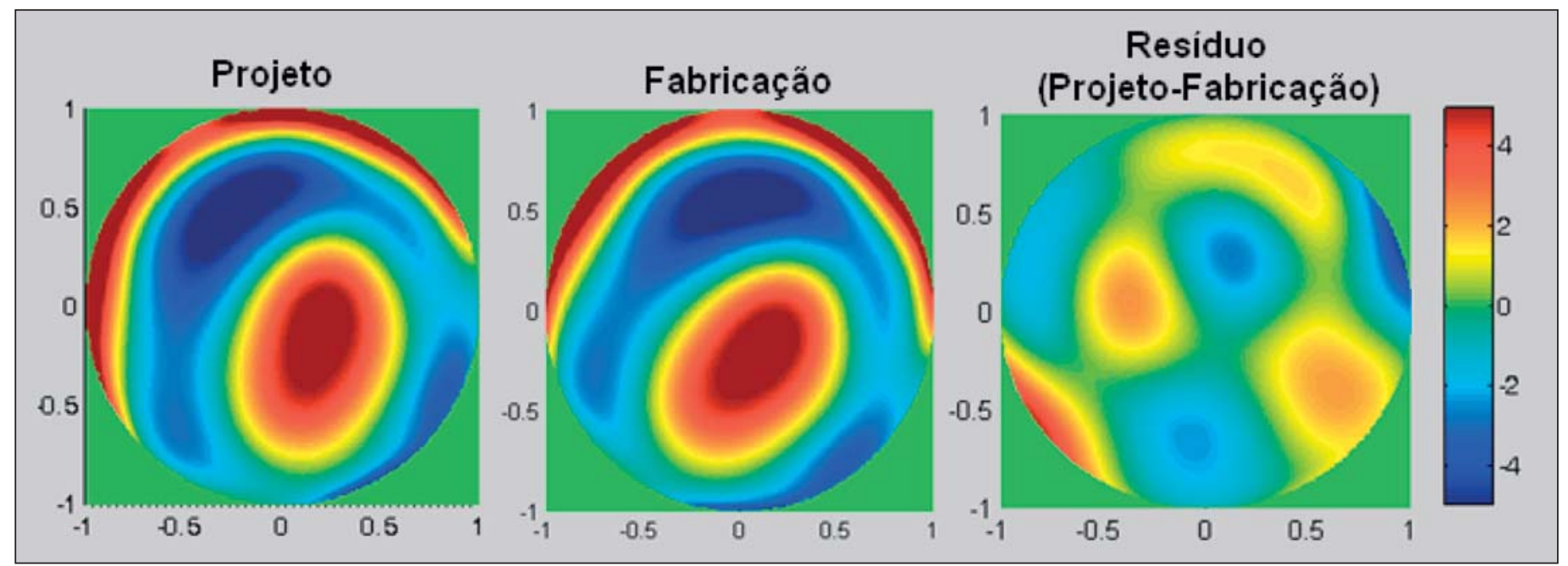

Figura 5 - Exemplo do resultado para lente projetada e fabricada para um paciente com ceratocone 


\begin{tabular}{|c|c|c|c|c|c|}
\hline $\begin{array}{l}\text { Coeficiente de Zernike } \\
\text { EQM (erro quadrático } \\
\text { médio) }=1,830741 \\
\text { EQM é referente aos coefici }\end{array}$ & entes de 1 a 65 & Medida 1 & Medida 2 & Medida 3 & Desvio padrão \\
\hline 0 & Pistão (não computado) & & & & \\
\hline 1 & Tilt no eixo $X$ & & & & \\
\hline 2 & Tilt no eixo $Y$ & & & & \\
\hline 3 & Desfocalização & & & & \\
\hline 4 & Astigmatismo (diag.) & & & & \\
\hline 5 & Astigmatismo & & & & \\
\hline 6 & Coma primário (eixo $\mathrm{X}$ ) & 0,312586 & 0,309461 & 0,31565 & 0,003095 \\
\hline 7 & Coma primário (eixo Y) & 0,698142 & 0,69116 & 0,677337 & 0,010588 \\
\hline 8 & Astigmatismo secund. (eixo y) & $-0,18244$ & $-0,18061$ & $-0,177$ & 0,002767 \\
\hline 9 & Astigmatismo secund. (eixo $x$ ) & $-0,05644$ & $-0,05588$ & $-0,05476$ & 0,000856 \\
\hline 10 & Aberração esférica & 0,050629 & 0,050122 & 0,04912 & 0,000768 \\
\hline 11 & & 0,280642 & 0,277836 & 0,283392 & 0,002778 \\
\hline 12 & & 0,120276 & 0,119074 & 0,121455 & 0,001191 \\
\hline 13 & & 0,134004 & 0,132664 & 0,135317 & 0,001327 \\
\hline 14 & & $-0,07403$ & $-0,07329$ & $-0,07475$ & 0,000733 \\
\hline 15 & Coma secundário (eixo x) & $-0,38126$ & $-0,37745$ & $-0,385$ & 0,003775 \\
\hline 16 & Coma secundário (eixo y) & $-0,01676$ & $-0,0171$ & $-0,01744$ & 0,000339 \\
\hline 17 & & $-0,11046$ & $-0,11267$ & $-0,11493$ & 0,002231 \\
\hline 18 & & 0,095094 & 0,096996 & 0,098936 & 0,001921 \\
\hline 19 & & $-0,00988$ & $-0,01008$ & $-0,01028$ & 0,0002 \\
\hline 20 & & $-0,12117$ & $-0,12359$ & $-0,12606$ & 0,002448 \\
\hline 21 & Aberração esférica secundária & 0,163309 & 0,166575 & 0,163244 & 0,001905 \\
\hline 22 & Astigmatismo terciário (eixo x) & $-0,09079$ & $-0,08989$ & $-0,08809$ & 0,001377 \\
\hline 23 & Astigmatismo terciário (eixo y) & 0,036571 & 0,036205 & 0,035481 & 0,000555 \\
\hline 24 & & 0,055077 & 0,054526 & 0,053435 & 0,000835 \\
\hline 25 & & 0,001774 & 0,001756 & 0,001721 & 2,69E-05 \\
\hline 26 & & 0,057255 & 0,056682 & 0,055548 & 0,000868 \\
\hline 27 & & $-0,06697$ & $-0,0663$ & $-0,06497$ & 0,001016 \\
\hline 28 & Coma terciário (eixo y) & 0,053488 & 0,052953 & 0,054012 & 0,00053 \\
\hline 29 & Coma terciário (eixo $\mathrm{x}$ ) & $-0,05697$ & $-0,0564$ & $-0,05753$ & 0,000564 \\
\hline 30 & & $-0,00155$ & $-0,00153$ & $-0,00156$ & $1,53 \mathrm{E}-05$ \\
\hline 31 & & 0,028889 & 0,029467 & 0,028877 & 0,000337 \\
\hline 32 & & $-0,07949$ & $-0,08108$ & $-0,07946$ & 0,000927 \\
\hline 33 & & 0,028009 & 0,028569 & 0,027998 & 0,000327 \\
\hline 34 & & $-0,02867$ & $-0,02924$ & $-0,02866$ & 0,000334 \\
\hline 35 & & 0,070358 & 0,071765 & 0,07033 & 0,000821 \\
\hline 36 & & 0,023157 & 0,02362 & 0,023148 & 0,00027 \\
\hline 37 & & $-0,0408$ & $-0,04162$ & $-0,04079$ & 0,000476 \\
\hline 38 & & $-0,02416$ & $-0,02464$ & $-0,02415$ & 0,000282 \\
\hline 39 & & $-0,05601$ & $-0,05713$ & $-0,05598$ & 0,000653 \\
\hline 40 & & 0,050718 & 0,051732 & 0,052767 & 0,001025 \\
\hline 41 & & $-0,05411$ & $-0,05357$ & $-0,05464$ & 0,000536 \\
\hline 42 & & 0,046516 & 0,046051 & 0,046972 & 0,000461 \\
\hline 43 & & 0,023481 & 0,023246 & 0,023711 & 0,000232 \\
\hline 44 & & $-0,04295$ & $-0,04252$ & $-0,04167$ & 0,000651 \\
\hline 45 & & $-0,01041$ & $-0,01031$ & $-0,0101$ & 0,000158 \\
\hline 46 & & 0,018214 & 0,018032 & 0,017671 & 0,000276 \\
\hline 47 & & 0,052411 & 0,051887 & 0,050849 & 0,000795 \\
\hline 48 & & $-0,02045$ & $-0,02024$ & $-0,01984$ & 0,00031 \\
\hline 49 & & 0,031301 & 0,030988 & 0,030368 & 0,000475 \\
\hline 50 & & $-0,03871$ & $-0,03833$ & $-0,03909$ & 0,000383 \\
\hline 51 & & 0,010124 & 0,010326 & 0,01012 & 0,000118 \\
\hline 52 & & 0,04351 & 0,04438 & 0,043493 & 0,000508 \\
\hline 53 & & $-0,03186$ & $-0,0325$ & $-0,03185$ & 0,000372 \\
\hline 54 & & 0,005736 & 0,005851 & 0,005734 & 6,69E-05 \\
\hline 55 & Aberração esférica quaternária & $-0,00417$ & $-0,00426$ & $-0,00417$ & 4,87E-05 \\
\hline 56 & & $-0,01289$ & $-0,01276$ & $-0,01301$ & 0,000128 \\
\hline 57 & & $-0,05357$ & $-0,05304$ & $-0,05198$ & 0,000813 \\
\hline 58 & & 0,023469 & 0,023235 & 0,02277 & 0,000356 \\
\hline 59 & & $-0,06213$ & $-0,06151$ & $-0,06028$ & 0,000942 \\
\hline 60 & & $-0,00932$ & $-0,00922$ & $-0,00904$ & 0,000141 \\
\hline 61 & & $-0,01057$ & $-0,01046$ & $-0,01025$ & 0,00016 \\
\hline 62 & & $-0,0274$ & $-0,02712$ & $-0,02658$ & 0,000416 \\
\hline 63 & & 0,035913 & 0,035554 & 0,034843 & 0,000545 \\
\hline 64 & & 0,018677 & 0,018491 & 0,018121 & 0,000283 \\
\hline 65 & & 0,01761 & 0,017434 & 0,017782 & 0,000174 \\
\hline
\end{tabular}


líndricas que permitem ótimos resultados. Como pode ser percebido os coeficientes têm valores bastante próximos para cada medida, o que demonstra a alta reprodutibilidade do equipamento. $\mathrm{O}$ desvio médio padrão para coeficientes individuais não passou de 0,10 micrômetro, sendo que o desvio médio entre o EQM de cada medida foi de apenas 0,04 micrômetro. Como as aberrações totais induzidas na lente estão na ordem de 4 micrômetros, podemos afirmar que a precisão do instrumento está em torno de $1 \%$ do valor da medida.

Para comprovar a eficiência da técnica, comparamos as medidas mostradas na figura 4 com o projeto inicialmente enviado para a B\&L. Na figura 5 podemos ver imagens relativas à frente de onda planejada (aquela que foi projetada baseando-se nas correções para o paciente), da lente de contato medida no aparelho e o resíduo entre aquilo que foi projetado e aquilo que foi fabricado pela empresa Bausch \& Lomb.

Os mapas com códigos de cor estão associados à elevação da frente de onda e portanto diretamente associados à intensidade de aberrações ópticas da lente de contato. Todas as aberrações mostradas aqui são de alta ordem, ou seja, a partir do sétimo termo (coma vertical) de Zernike até o sexagésimo sexto termo ${ }^{(6-7)}$.

\section{DISCUSSÃO}

Analisando os valores descritos na tabela 1 e pelas figuras 4 e 5, observou-se que a instrumentação óptica desenvolvida neste trabalho foi capaz de medir as aberrações ópticas de alta ordem com precisão. Na figura 3 há um gráfico com três medidas consecutivas para a mesma lente, que foi re-inserida na célula úmida a cada medida. O erro quadrático médio foi de 0,04 micrômetro, sendo o valor médio de EQM para olhos emétropes de 0,5 micrômetro. Este valor está próximo ao ruído de fundo do instrumento utilizado para as medidas $(0,03$ micrômetro). Na figura 5 pode-se notar a diferença entre aquilo que foi projetado e aquilo que foi fabricado, notando-se uma diferença pequena, mas detectável pelo aparelho construído.

\section{CONCLUSÃO}

Pode-se concluir que o aparelho desenvolvido aqui foi suficientemente preciso para o objetivo ao qual está sendo destinado. Este instrumento é parte de um projeto em andamento, ou seja, a confecção de LCP para correção eficiente de aberrações de alta ordem. Por este motivo foram mostrados os resultados obtidos para a lente de um paciente com ceratocone.

\section{ABSTRACT}

Purpose: Soft contact lenses with spherical base curves have been used for many years. The computation of the refractive powers of these lenses is easy, requiring only that one uses the lens maker equation for thick lenses. Nevertheless, for customized contact lenses, there is yet no reliable method for measuring the higher order optical aberrations. In this study we have developed in the Center for Visual Sciences of the University of Rochester an optical apparatus that allows for precise measurement of low and high order aberrations of customized soft contact lenses. Methods: An optical apparatus was mounted on a conventional optical bench. This apparatus consists of a wet cell where the contact lenses are placed, a series of relay lenses, mirrors, beam splitters, and a Hartmann-Shack sensor. Bausch \& Lomb manufactured the lenses used in this study. Results: The root mean square error (RMSE) of the instrument was 0.04 microns. Given that the RMSE of the customized lens is between 4 and 6 microns, i.e., the precision of the instrument is approximately $1 \%$. This precision is more than sufficient for the type of measurements necessary for manufacturing customized contact lenses. Conclusion: The instrument developed is extremely precise for measuring high order aberrations - up to the 10th order Zernike polynomials, that is, up to the $66^{\text {th }}$ term. This technology is important for the development of new methods of optical corrections for patients that usually do not adapt to normal sphere-cylinder spectacles or that cannot undergo refractive surgery, such as those which have keratoconus, for example.

Keywords: Corneal topography; Lenses, intraocular; Refraction, ocular; Contact lenses, hydrophilic; Keratoconus

\section{REFERÊNCIAS}

1. Moreira SMB, Moreira H. Lentes de contato. 2a ed. São Paulo: Cultura Médica; 1998.

2. Mandel RB. Contact lens practice. 4th ed. Springfield, Illinois; Charles C. Thomas; 1988.

3. Carvalho LA, Corzine JC, Miller KL, Carney T, Klein SA, Polse KA. A computer-based image processing system for assessment of contact lens translation and rotation, In: ARVO (The Association for Research in Vision and Ophthalmology). Meeting. Fort Lauderdale, Florida -May 9-14, 1999.

4. Carvalho LA, Yoon G. Costumized Contact lenses for correction of higher order aberrations on a keratoconic patient. In: ARVO (The Association for Research in Vision and Ophthalmology). Meeting, Fort Lauderdale, Florida May, 2006. In press.

5. Carvalho LA. A simple and effective algorithm for detection of arbitrary Hartmann-Shack patterns. J Biomed Inform. 2004;37(1):1-9.

6. Carvalho LA. Absolute accuracy of Placido-based videokeratographs to measure the optical aberrations of the cornea. Optom Vis Sci. 2004;81(8):616-28.

7. Carvalho LA. Accuracy of Zernike polynomials in characterizing optical aberrations and the corneal surface of the eye. Invest Ophthalmol Vis Sci. 2005; 46(6):1915-26.

\section{Ao enviar um artigo para publicação,}

leia ATENTAMENTE as instruções para autores, constante no final de cada fascículo. 